ORIGINAL PAPER

Petra Deprés-Brummer · Moshe Itzhaki

Piet J. M. Bakker · Frans J. Hoek

Kees H. N. Veenhof · Ronald de Wit

\title{
The usefulness of CA15.3, mucin-like carcinoma-associated antigen and carcinoembryonic antigen in determining the clinical course in patients with metastatic breast cancer
}

Received: 11 July 1994/Accepted: 27 February 1995

\begin{abstract}
Levels of mucin-like carcinoma-associated antigen (MCA), CA15.3 and carcinoembryonic antigen (CEA) were measured in consecutive serum samples of 40 women with metastatic breast cancer. A change in antigen level of more than $25 \%$, either an increase or a decrease, was considered to predict progressive or responsive disease respectively. A change of less than $25 \%$ was considered to predict stable disease. MCA, CA15.3 and CEA were elevated in the serum of $68 \%$, $76 \%$ and $48 \%$ of the patients respectively $(P<0.05)$. The overall prediction of clinical course was similar for all three markers. A more than $25 \%$ increase of MCA, CA15.3, and CEA was observed in $61 \%, 54 \%$ and $36 \%$ respectively. The predictive value of a more than $25 \%$ increase was high for all three markers: $94 \%, 94 \%$, $83 \%$. Changes in marker levels were correlated with each other. Logistic regression analysis showed that combining MCA and CA15.3 did not improve the prediction further. In conclusion, these tumour markers may help in evaluating the disease course and there is no advantage in combining $\mathrm{MCA}$ and CA15.3.
\end{abstract}

P. Deprés-Brummer · M. Itzhaki

Laboratoire de "Rythmes Biologiques et Chronotherapeutique". I.C.I.G., Hôpital Paul Brousse, France

P. J. M. Bakker - K. H. N. Veenhof

Department of Medical Oncology, Academic Medical Centre, Amsterdam, The Netherlands

F. J. Hoek

Department of Clinical Chemistry, Academic Medical Centre, Amsterdam, The Netherlands

R. de Wit (बব)

Department of Medical Oncology, Rotterdam Cancer Institute/ Dr. Daniel den Hoed Kliniek, P.O. Box 5201, 3008 AE Rotterdam, The Netherlands

Fax: + 31-10-4851618
Key words Breast cancer - MCA - Tumour markers - CEA · CA15.3

\section{Introduction}

Carcinoembryonic antigen (CEA) is a widely used tumour marker in monitoring patients with advanced metastatic breast cancer (Beard and Haskell 1986; Bieglmayer et al. 1989). Changes in elevated CEA levels correlate with changes in disease activity in $33 \%-85 \%$ of patients, the higher percentages having cut-off levels above $20 \mathrm{ng} / \mathrm{ml}$ (Beard and Haskell 1986; Colomer et al. 1989; Loprinzi et al. 1986; Haagensen et al. 1978; Tormey and Waalkes 1978; Waalkes et al. 1984). However, its use is limited by low sensitivity. Elevated levels vary from $35 \%$ to $70 \%$ in different series (Bieglmayer et al. 1989; Haagensen et al. 1978; Tormey and Waalkes 1978; Waalkes et al. 1984). Routine use of the CEA assay has been discouraged with the contention that it does not lead to more effective treatment (Fletcher 1986). Recently two new immunoassays for breast cancer have been developed using CA15.3 and mucin-like carcinoma-associated antigen (MCA) (Bieglmayer et al. 1989; Hayes et al. 1986). The incidence of elevated CA15.3 levels in patients with metastatic breast cancer has been reported to be as high as $80 \%$ and therefore this marker may be of value in monitoring the clinical course (Hayes et al. 1986). Correlation with clinical course was found to vary from $60 \%$ to $75 \%$ (PonsAnicet et al. 1987; Tondini et al. 1988).

MCA has also been reported to be a sensitive marker for breast carcinoma. Sensitivity and specificity appear comparable with those of CA15.3 (Bieglmayer et al. 1989; Linsley et al. 1988; Stähli et al. 1988; de Wit et al. 1991; Miserez et al. 1991). In order to determine whether these two newly developed markers are more suitable for monitoring, we examined CEA, MCA and CA15.3 levels in the serum of patients with advanced breast cancer and related changes to the clinical course. 


\section{Materials and methods}

Sample collection

Serum samples of a consecutive series of patients with metastatic breast cancer were collected. These samples were drawn at the time of clinical tumour evaluation. Appropriate marker values were only considered if sufficient data on treatment response during that period and follow-up were available. Evaluable patients were defined as having measurable or evaluable metastatic disease and a minimum of two serum samples with an interval of at least 1 month.

Criteria of evaluation

Measurement of disease course (response, progression, or stable disease) was determined by clinical examination, plain radiographs, computer-aided tomography, ultrasonography, and bone scans. A response required a more than $50 \%$ decrease in size of measurable or evaluable lesions, whilst disease progression required an increase of $25 \%$ or the appearance of new lesions. Stable disease was defined as neither response nor progression of disease. Pleural effusions and changes in liver function tests were not by themselves considered evaluable criteria. In the presence of osseous metastases as the only available disease parameter, progression required the appearance of new lesions on serial bone scans or new osteolytic lesions on radiographs, whereas a response required both the disappearance or decrease of isotope uptake on a bone scan combined with at least some other evidence such as a decline in serum alkaline phosphate, recalcification of osteolytic lesions and improvement of the patient performance status.

\section{Determination of tumour marker levels}

Enzyme immunoassays were used to determine MCA (Hoffman-la Roche, Basel, Switzerland), CA15.3 (CIS, Gif sur Yvette-Cedex, France) and CEA (Abbot, Amstelveen, The Netherlands) levels. Values above the 99th percentile of the normal value distribution were considered elevated: MCA $16 \mathrm{U} / \mathrm{ml}, \mathrm{CA} 15.330 \mathrm{U} / \mathrm{ml}$ and CEA $5 \mathrm{ng} / \mathrm{ml}$. All samples were measured twice.

\section{Analysis of Results}

Changes of more than $25 \%$ from the initial marker serum concentrations were arbitrarily defined as significant. To avoid multiplemeasurement bias by repetitive measurements in cases of concordant observations, only the first two blood samples were used for the analysis. Sensitivity and specificity and positive predictivue values of changes in serum concentrations were calculated. The clinical course was divided into progressive or non-progressive disease, the latter comprising both responsive and stable disease. To qualify for the category of non-progressive disease during the observation period, it was required that this condition was maintained for at least 3 months after the second measurement. This excluded the possibility that marker changes were indicative of disease progression before this was clinically detectable. Sensitivity was defined as the proportion of the people with progressive disease and an increase of their marker level of more than $25 \%$. Specificity was defined as the proportion of the people with non-progressive disease and a marker level increase less than $25 \%$. Predictive value was defined as the proportion of patients with a more than $25 \%$ increase who had progressive disease.
Statistical analysis

The $\chi^{2}$-test was used to compare the percentages of elevations of the three markers. The Wilcoxon test was used to explore the relation between the first and second value of each marker. The Spearman test was used to examine the correlation between course of disease (response, progressive disease, stable disease) and the difference in marker level, which was classified as 1 for a $25 \%$ decrease, 2 for a less than $25 \%$ decrease or increase and 3 for a more than $25 \%$ increase of the initial marker level. The Spearman test was also used to examine the correlation between the differences in the different markers. Logistic regression analysis was used to test the efficacy of the three tests.

\section{Results}

\section{Patient characteristics}

A consecutive series of 76 patients with metastatic breast cancer was examined; 40 patients had both measurable or evaluable disease, and two appropriate serum samples. Nineteen patients were excluded because only one serum sample was available, and 17 patients had no measurable or evaluable disease. Of the 40 patients who were included in the analysis, the mean age was $63.9 \pm 13.4$ years. Twenty-seven patients received hormonal treatment, 8 patients received chemotherapy and 5 patients received no antitumour therapy. At the time of the second serum sample 28 patients were deemed to have progressive disease and 12 patients had non-progressive disease, of whom 5 applied for objective tumour regression, and 7 had stable disease.

Nineteen patients had bone metastases predominantly, 12 patients had visceral metastases, and 9 patients had both extensive bone and visceral metastases; $65 \%$ of all patients had two or more metastatic sites. The mean interval between follow-up samples was $4.5 \pm 3.4$ months.

\section{Comparison of circulating MCA, CA15.3 and CEA levels}

MCA and CA15.3 were elevated in the serum of $67.5 \%$ and $75.6 \%$ of patients respectively, whereas CEA was elevated in $47.5 \%$ of patients. This difference was statistically significant (overall $\chi^{2}=7.04, P<0.05$; CEA versus MCA $\chi^{2}=3.2, P=0.1$; CEA versus CA 15.3 $\left.\chi^{2}=6.4, P=0.05\right)$. In 16 cases $(40 \%)$ all three markers were elevated, in 15 cases MCA and CA15.3 were positive whereas CEA was negative $(37.5 \%)$ and in 7 cases $(17.5 \%)$ only one marker was elevated. Table 1 shows the data on sensitivity, specificity and positive predictive values. The sensitivity of a more than $25 \%$ increase of MCA, CA15.3 and CEA was $60.7 \%$, $53.6 \%$ and $35.7 \%$ respectively $\left(\chi^{2}=3.7, P=0.1\right)$. The predictive value of a more than $25 \%$ increase was high (but not statistically different) for all three markers $(94.4 \%, 93.8 \%$ and $83.3 \%$ respectively). 
Table 1 Sensitivity, specificity and positive predictive values of more than $25 \%$ changes in serum marker levels

\begin{tabular}{lllllll}
\hline Evaluation & MCA & \multicolumn{3}{l}{ CA15.3 } & \multicolumn{2}{l}{ CEA } \\
\hline Sensitivity & $60.7 \%$ & $(17 / 28)$ & 53.6 & $(15 / 28)$ & 35.7 & $(10 / 28)$ \\
Specificity & $91.7 \%$ & $(11 / 12)$ & 90.0 & $(9 / 10)$ & 83.3 & $(10 / 12)$ \\
$\chi^{2}=0.44$ NS \\
Positive predictive value & $94.4 \%$ & $(17 / 18)$ & 93.8 & $(15 / 16)$ & 83.3 & $(10 / 12) \chi^{2}=0.86 \mathrm{NS}$ \\
\hline
\end{tabular}

Clinical course was correctly predicted by MCA in 24 cases, by CA15.3 in 22 cases and by CEA in 17 cases. Changes in MCA levels were correlated with changes in CA15.3 and CEA levels (MCA and CA 15.3 $r=0.35, P=0.03$; MCA and CEA $r=0.34, P=0.03$ ). Changes in CA15.3 levels were also correlated with changes in CEA levels $(r=0.34, P=0.037)$. The course of disease was highly correlated with a change in marker level of MCA $(r=0.53, P=0.0004)$ and CA15.3 $(r=0.44, P=0.0006)$, but less strongly with CEA $(r=0.32, P=0.044)$.

Analysis of paired observations revealed that the association of marker level changes in MCA, CA15.3 and CEA showed three identical patterns in only 8 of the 40 patients, whereas in 8 cases none correlated with clinical course. The association of both MCA and CA15.3 (concordant observations) correctly predicted the clinical course in 16/40 cases, CA15.3 and CEA correctly predicted the clinical course in 11 /40 cases, and for MCA and CEA it was $9 / 40$ cases. To analyse further the relationship between clinical change and markers a logistic regression analysis was used. As dependant variable the clinical course was divided into progression against non-progression and response against non-responsive disease.

MCA and CA15.3 were found to contribute to the model significantly only in the first comparison (progression against non-progression; $P=0.037$ and $P=0.046$ respectively). Combining the two markers did not contribute further. In the second comparison (responsive against non-responsive disease), only CEA was a significant contributor $(P=0.026)$.

\section{Discussion}

The serum levels of MCA, CA15.3 and CEA were compared for their clinical usefulness in the management of patients with metastatic breast cancer. Of 40 patients included in the analysis, MCA, CA15.3 and CEA were above the 99th percentile of the normal value distribution in at least one serum sample in $75 \%$, $84 \%$ and $55 \%$ of the cases respectively (overall $\chi^{2}=8.5, P<0.02 ;$ CEA versus MCA $\chi^{2}=3.5 P=0.1$; CEA versus CA15.3 $\chi^{2}=7.8, P=0.05$ ). Relative changes of MCA, CA15.3 and CEA levels were found to be correlated. When relative changes of these markers were correlated with disease course, the predictive value of a change of $25 \%$ or more in one of these markers proved to be high for all three markers: $94.4 \%$, $93.8 \%$ and $83.3 \%$, for MCA, CA15.3 and CEA respectively. The sensitivity of these markers was poor: MCA $60.7 \%$, CA15.3 53.6\%, CEA $35.7 \%$ with a tendency to higher sensitivity for both MCA and CA15.3. MCA and CA 15.3 were highly correlated, and logistic regression analysis demonstrated that a combination of MCA and CA15.3 did not further improve the prediction. Our results indicate that, if either MCA, CA15.3 or CEA is elevated in the serum of a patient with metastatic breast cancer, a change of $25 \%$ or more accurately predicts the disease course in at least $85 \%$ of cases. Our results are in accord with previous reports suggesting that these novel serum markers, such as CA15.3, are superior to CEA for monitoring (Hayes et al. 1986; Tondini et al. 1988).

Breast cancer often metastasizes to the skeleton and is the dominant feature of advanced disease in a considerable number of patients. In these cases, the disease course is evaluated by modestly sensitive criteria such as new lesions on a bone scan, the size of osteolytic lesions, evidence of recalcification on X-rays and blood chemistry results such as alkaline phosphatase and clinical performance. Also in our series of 76 patients, despite our acceptance of such criteria when concordant for evaluating disease, 17 patients, the majority of whom received hormonal treatment, were excluded from analysis because their disease course could not properly be evaluated. The sensitivity and predictive value of MCA and CA15.3 are sufficiently high to be of use in patients who do not have measurable and/or evaluable lesions. However, because of the observed discordances between marker pattern and objective disease course, these marker changes should be considered indicative, and cannot replace disease evaluation by conventional methods.

Although MCA and CA15.3 have been developed against different epitopes of breast tumour cells, we have previously reported that these markers identify nearly the same patients (de Wit et al. 1991). Bieglmayer et al. (1989) also found a similar correlation between these two tumour markers. On the basis of their results from binding tests with the antibodies used in the MCA and CA15.3 assays, they concluded that the monoclonal antibodies b-12 (MCA assay) and 115D8/DF3 (CA15.3 assay) recognize coexisting epitopes on mucin-like antigens that belong to a number of glycoproteins suitable for tumour monitoring. This 
study demonstrates that the correlation of CA15.3 and MCA serum levels with disease course is also nearly identical. Therefore, either of these newly developed markers can be used, and there is no advantage in combining the two markers.

Acknowledgement We thank Mrs. J.J. Verburg for the analysis of the marker levels, and Miss Therèse van Eijk for her help with the final layout of the manuscript.

\section{References}

Beard DB, Haskell CM (1986) Carcinoembryonic antigen in breast cancer, clinical review. Am J Med 80:40-245

Bieglmayer C, Szepesi T, Neunteufel W, Nowotny C (1989) MCA, a monoclonal-antibody-defined breast tumor associated antigen and its relation to CA15.3. Tumor Biol 10:232-242

Colomer R, Ruibal A, Salvader L (1989) Circulating tumor marker levels in advanced breast carcinoma correlate with the extent of metastatic disease. Cancer 64:1674-1682

Fletcher RH (1986) Carcinoembryonic antigen. Ann Intern Med 104:66-73

Haagensen DE, Kister SJ, Vandevoorde JP, Gates JB, Smart EK, Hansen HJ, Wells SA (1978) Evaluation of carcinoembryonic antigen as a plasma monitor for human breast carcinoma. Cancer 42:1512-1519

Hayes DF, Zurawski VR, Kufe DW (1986) Comparison of circulating CA15.3 and carcinoembryonic antigen levels in patients with breast cancer. J Clin Oncol 4:1542-1550
Linsley PS, Brown JP, Magnani JC, Horn D (1988) Monoclonal antibodies reactive with mucin glycoproteins found in sera from breast cancer patients. Cancer Res 48:2138-2148

Loprinzi CL, Tormey DC, Rasmussen P, Falkson G, Davis TE, Falkson HC, Chang AYC (1986) Prospective evaluation of carcinoembryonic antigen levels and alternating chemotherapeutic regimens in metastatic breast cancer. $\mathrm{J}$ Clin Oncol 4:46-56

Miserez AR, Günez I, Müller-Brand J, Walther E, Fridrich R, Macke $H$ (1991) Clinical value of a mucin-like carcinoma-associated antigen in monitoring breast cancer patients in comparison with CA 15-3. Eur J Cancer 27:126-131

Pons-Anicet DMF, Krebs BP, Mira R, Namer M (1987) Value of CA15.3 in the follow up of breast cancer patients. Br J Cancer 55:567-569

Stahli C, Caravatti M, Aeschbacher M, Kocyba C, Takacs B, Carmann $H$ (1988) Mucin like carcinoma associated antigen defined by three monoclonal antibodies against different epitopes. Cancer Res 48:6799-6802

Tondini C, Hayes DF, Gelman R, Henderson IC, Kufe DW (1988) Comparison of $\mathrm{CA} 15.3$ and carcinoembryonic antigen in monitoring the clinical course of patients with metastatic breast cancer. Cancer Res 48:4107-4112

Tormey DC, Waalkes TP (1978) Clinical correlation between CEA and breast cancer. Cancer 42:1507-1511

Waalkes TP, Enterline JP, Sharper JH, Abeloff MD, Ettinger DS (1984) Biological markers for breast carcinoma. Cancer 53:644-651

Wit R de, Bakker PJM, Hoek FJ, Veenhof CHN (1991) The value of MCA, CA15-3, CEA and CA125 to discriminate between metastatic breast cancer and metastatic adenocarcinoma of other primairy site. J Intern Med 229:463 466 